\title{
Hommage à Jean-Pierre Chartier
}

\begin{abstract}
T ean-Pierre Chartier, un "Professeur Espiègle », selon les mots de celles qui furent ses élèves, vient de nous quitter. Un départ qui s'est fait dans une discrétion qui ne pouvait lui convenir, lui qui aimait que les choses soient dites, qui suggérait que le savoir se partage et que la culture ne soit pas un héritage, mais qu'elle doive se conquérir. Cet engagement, au cœur de sa démarche est lisible dans ses quatorze ouvrages et ses trente huit articles scientifiques conceptualisant ses recherches. C'est celui d'un praticien chercheur comme il aimait à le dire. Car on ne s'approprie pas Jean-Pierre Chartier, cette carrure de sportif qui paraissait se suffire à elle-même avec sa moto et son casque, assez éloignée des guirlandes qui s'allument et qui s'éteignent et des ors de l'université dont il ne méconnaissait pas l'importance pour autant.
\end{abstract}

Docteur en psychologie, docteur d'État en psychologie clinique (ancien régime) membre du $I V^{\mathrm{e}}$ groupe qu'il incarnait assez bien quand il partait sur les chemin de "Translacanie ", là, il savait se montrer intarissable, plein d'humour et surtout lucide à propos du trépied de son appartenance: F. Perrier; P. Aulagnier (son pictogramme), et J.-P. Valabrega. Les membres fondateurs, produits de dissidence. Mais Jean-Pierre était de formation psychologique, promotion 67 de psycho-prat comme on disait alors, une École des Psychologues Praticiens qui dû mener des luttes pour trouver un équilibre avec l'Université. Et sur ce terrain, alors que j'étais responsable des stages â l'UER Institut de Psychologie, et lui directeur de l'École, nous partagions cette conviction que les Institutions de la Société Civile comme son École défendaient des valeurs de la République peut-être un peu trop délaissées par ses propres institutions. Jean-Pierre Chartier, un Républicain ! Surtout un homme chaleureux qui parlait avec tendresse, alors qu'il n'aimait pas trop les atermoiements, de Lisette Fanchon ou de l'abbé Besson, les fondateurs de l'École dont il avait pris la succession. Cet après-midi nous discutions des livres de l'abbé bradés sur la brocante du boulevard Auguste Blanqui dans le XIII ${ }^{\mathrm{e}}$ arrondissement de Paris ou du "Congrès Touati » à Strasbourg, et du concert dans la cathédrale. Souvenirs parcellaires, lapidaires. Chacun peut en faire état. C'est toute la singularité du professeur espiègle d'avoir su créer des complicités dans lesquelles son nœud papillon peut avoir joué un rôle. Depuis ce 20 février, Jean Pierre Chartier repose à Montparnasse.

Il est intéressant de brosser l'itinéraire de ce praticien chercheur. On doit le faire sans oublier de l'inscrire dans une tradition: J. Chazal, celle du droit et de ma 
génération; celle de l'Éducation Surveillée et de la Protection Judiciaire de la Jeunesse (PJJ), celle aussi des travaux du Centre de Recherche de Vaucresson. Qu'on se souvienne du temps de la fermeture du J 3 de la maison d'arrêt de Fresnes (les mineurs du temps de Mme de Gentile et de Y. Roumajon), de la création du Centre des Jeunes Détenus (CJD) de Fleury Mérogis et de son ouverture (1972) contemporaine de la création par le Docteur Y. Roumajon, du Premier Centre pour psychopathes de Bois Maison à Igny, Vauhallan d'inspiration Canadienne (le Boscoville du père N. Mailloux et du Pr M. Lemay, notre Breton d'origine) en lien avec la colonie de Perray-Vaucluse (Mme le Dr F. Grasset-Coste). C'était une période féconde entre justice et psychologie. Autant d'aspects qui allaient conduire le psychologue-psychanalyste Jean-Pierre Charlier à envisager la création d'un dispositif pour accueillir et se confronter avec ceux qu'il appellera avec bienveillance " les chevaliers de Thanatos ", des chevaliers qui ouvriront une approche nouvelle de la violence et du corps à corps, une violence pensée comme pathologique et envisagée comme pouvant se prêter à des stratégies thérapeutiques (ce qui peut paraître aujourd'hui comme élémentaire ne l'était guère hier). Il faut rendre ici hommage à l'École de Strasbourg et à R. Helbrunn dont les travaux, et l'engagement dans la confrontation avec le corps ont considérablement transformé cette période de l'évolution humaine, cette période définie par le Professeur M. Debesse comme celle du « n'être plus et pas encore... ». Une période qui allait favoriser la rencontre entre le Professeur H. Flavigny, du service adolescent de l'Hopital International Universitaire du Boulevard Jourdan, les membres des Équipes Amitiés animées par Marie Thérèse Flavigny, et Jean-Pierre Chartier qui fut choisi comme le psychanalyste de l'équipe avec pour mission bien comprise de

" remanier sans cesse nos modes d'approches et de réponses pour l'adapter aux plus jeunes en perpétuelle métamorphose, rechercher aussi leurs potentialités ", un objectif qui traversera ses ouvrages à venir.

Le lecteur désireux de se familiariser avec l'œuvre de Jean-Pierre Chartier commencera la lecture de son ouvre par un de ses livres les plus aboutis, soit Les adolescents difficiles, paru en 2011 chez Dunod.

On comprend mieux l'orientation criminologique que le professeur J.-P. Chartier donnera à l'École des Psychologues Praticiens, son lieu d'origine.

Serge G. Raymond et Olivier Douville 